\title{
EVOLUTION OF STATES OF AN INFINITE PARTICLE SYSTEM WITH NONLOCAL BRANCHING
}

\author{
YURI KOZITSKY AND AGNIESZKA TANAŚ
}

\begin{abstract}
We study the evolution of states of an infinite system of point particles dwelling in a locally compact Polish space $X$. Each particle produces at random a finite 'cloud' of offsprings distributed over $X$ according to some law, and disappears afterwards. The system's states are probability measures on an appropriate space of locally finite counting measures on $X$. Their evolution is obtained by solving the corresponding Fokker-Planck equation. We prove that this equation has a unique solution and discuss some of its properties. Our pivotal idea of dealing with infinite systems consists in passing to tempered counting measures by imposing appropriate restrictions on the branching. In this approach, we first solve a nonlinear evolution equation in the space of bounded continuous functions on $X$ - so called log-Laplace equation. Next we solve the Kolmogorov equation which is then used to solve the Fokker-Planck equation and thus describe the evolution in question.
\end{abstract}

\section{INTRODUCTION}

In recent years, the stochastic evolution of infinite particle systems attract considerable attention, see, e.g., [13, 14, 16, 17]. A related popular topic is measure-valued stochastic branching characterizing the evolution of random 'clouds' [8, 11, 19, see also [2, 3, 9, 15] and the literature quoted in these works. Let $X$ be a locally compact Polish space, $\mathcal{B}(X)$ its Borel $\sigma$-field and $\mathcal{N}$ be the set of all finite counting measures on $X$, i.e., $\nu(\Delta)$ is a nonnegative integer for each $\nu \in \mathcal{N}$ and $\Delta \in \mathcal{B}(X)$. It is known that the weak topology makes $\mathcal{N}$ a Polish space. By [6, Proposition 9.1.III, page 4] it follows that $\nu$ can be presented in the form $\nu=\sum_{i} \delta_{x_{i}}$, where $\delta_{x_{i}}$ are Dirac's measures and some of $x$ 's may coincide. By this formula one may interpret $\nu$ as a 'cloud' of particles located at points $x_{i} \in X$. The key aspect of this interpretation is that $\nu(X)$ - the total number of particles is finite as $\nu$ is a finite measure. Since in the course of branching each particle is replaced by a finite number of offsprings, the system remains finite during all its lifetime. Our main challenge in this work is to find out how to describe branching in an infinite particle system, in which the number of offsprings appearing at a given moment of time can be infinite. Along with a purely mathematical meaning of this question, its application value is related to the known fact that the collective behavior of a macroscopically large system can be understood only in the 'infinite system limit', see, e.g., [22, pages 5,6].

The way of dealing with an infinite particle system which we propose in this work has the following main aspects. Our object is an infinite collection of branching point particles - an infinite cloud - placed in a locally compact Polish space $X$ in such a way that each compact $\Lambda \subset X$ contains only finitely many elements of the cloud. This means that the corresponding counting measure belongs to the set of all locally finite counting measures $\mathcal{N}^{\#}$ and may take infinite values. The branching mechanism is described by a probability kernel $b$, i.e., a map $(X, \mathcal{B}(\mathcal{N})) \ni(x, \Xi) \mapsto b_{x}(\Xi) \in[0,1]$ such that each $b_{x}$ is a probability measure on $\mathcal{N}$ and $x \mapsto b_{x}(\Xi)$ is measurable for each Borel subset $\Xi$ of the set of all finite counting measures $\mathcal{N}$. Let $\delta(x)$ be the probability that a point at $x$ disappears without

2020 Mathematics Subject Classification. 35Q84; 37A50; 60J80; $93 \mathrm{E} 03$.

Key words and phrases. Polish space, evolution equation; branching; random counting measure; FokkerPlanck equation. 
leaving offsprings. That is, $\delta(x)=b_{x}\left(\Gamma^{0}\right)$, where $\Gamma^{0}$ is the singleton consisting of the zero measure. Our pivotal idea is to impose the condition that $1-\delta(x)$ vanishes at infinity, i.e., $1-\delta(x)<\varepsilon$ whenever $x \in \Lambda_{\varepsilon}^{c}:=X \backslash \Lambda_{\varepsilon}$, for a sufficiently big compact $\Lambda_{\varepsilon} \subset X$. Then we consider only those $\nu \in \mathcal{N}^{\#}$ - 'tempered measures' - for which $1-\delta(x)$ is integrable. Note that imposing a condition of this kind seems inevitable as an infinite system of branching particles can produce simultaneously an infinite cloud of offsprings that collapses into a compact $\Lambda \subset X$, and thus destroys the aforementioned local finiteness of the cloud. The same problem arises also in the dynamical theory of infinite systems of physical particles, see [10, page 223], where it is settled by imposing similar restrictions.

In dealing with particle systems, it is more convenient for us to stick at the 'corpuscular' terminology, i.e., to speak of configurations of particles instead of counting measures. Following [18], by a configuration $\gamma$ we mean a countable collection of point particles placed in $X$, where each particle is completely characterized by its location $x \in X$. Multiple locations are possible and each compact $\Lambda \subset X$ may contain only finitely many elements of $\gamma$. That is, 'configuration' is a more rigorous synonym of the aforementioned 'cloud'. The set of all configurations is denoted by $\Gamma$. Note that particles with the same location are indistinguishable, and there can only be finitely many of them located at a given $x$. By writing $\gamma \cup x$ we mean the configuration with added particle located at $x$. Likewise we define $\gamma \backslash x$ for $x \in \gamma$. Then by $\sum_{x \in \gamma}$ we mean $\sum_{i}$ for a certain enumeration of the elements of $\gamma$, cf. [18]. In this context, each $\nu \in \mathcal{N}^{\#}$ is presented as $\sum_{x \in \gamma} \delta_{x}$, which establishes a bijection between $\Gamma$ and $\mathcal{N}^{\#}$, see above. Typically, $\mathcal{N}^{\#}$ is equipped with the vague (weak-hash) topology which is the weakest topology that makes continuous the maps $\nu \mapsto \int_{X} g d \nu=: \nu(g)$ with all choices of compactly supported continuous functions $g: X \rightarrow \mathbb{R}$. Then the same topology on $\Gamma$ is defined by the maps $\gamma \mapsto \sum_{x \in \gamma} g(x)$. This makes $\Gamma$ and $\mathcal{N}^{\#}$ Polish spaces, see [6. Proposition 9.1,IV, page 6].

Our model is defined by the Kolmogorov operator

$$
(L F)(\gamma)=\sum_{x \in \gamma} \int_{\Gamma}[F(\gamma \backslash x \cup \xi)-F(\gamma)] b_{x}(d \xi),
$$

where $F$ is a suitable (test) function and $b$ is the aforementioned branching kernel. Note that the sum in (1.1) is infinite for infinite $\gamma$. The Kolmogorov operator (1.1) describes the distribution of the offsprings (constituting cloud $\xi$ ) of a particle located at $x$. Its detailed properties are listed in Assumption 2.5 below. By means of this $L$ we introduce the Kolmogorov equation

$$
\frac{d}{d t} F_{t}=L F_{t},\left.\quad F_{t}\right|_{t=0}=F_{0},
$$

describing the evolution of test functions, and the Fokker-Planck equation

$$
\mu_{t}(F)=\mu_{0}(F)+\int_{0}^{t} \mu_{s}(L F) d s
$$

that describes the evolution of states of the considered system. Here the states are probability measures on $\Gamma$; the set of all such states is denoted by $\mathcal{P}(\Gamma)$. Finally, $\mu(F):=\int F d \mu$ for suitable $F: \Gamma \rightarrow \mathbb{R}$. A comprehensive theory of the evolution equations of this kind can be found in [5].

The main steps in solving (1.3) undertaken in this work can be outlined as follows. In view of the form of $L$ given in (1.1), a natural way of solving (1.2) is to assume that $F_{t}$ has the following form

$$
F_{t}(\gamma)=\prod_{x \in \gamma} \phi_{t}(x)
$$

where, for each fixed $t, \phi_{t}$ is a continuous functions of $x \in X$ taking values in $(0,1)$. It turns out that the Kolmogorov equation admits solutions of this type with $\phi_{t}$ satisfying 
a nonlinear evolution equation derived from (1.2). In the theory of branching processes, such equations are called 'log-Laplace', see [8, pages 60,61]. We show that the latter has a unique solution and thereby obtain solutions of (1.2) in the Banach space of bounded continuous functions $F$, where $L$ is defined as a closed linear operator with a suitable domain $\mathcal{D}(L)$. Having this done, we show that (1.3) has a solution $t \mapsto \mu_{t}$ with $F \in \mathcal{D}(L)$. It turns out that the latter is big enough so that the following holds: (a) for each bounded measurable function $F^{\prime}, \mu\left(F^{\prime}\right)$ can be approximated by $\mu(F), F \in \mathcal{D}(L)$; (b) the mentioned solution be unique. The main result of this work is Theorem 4.2 that states that (1.3) has a unique solution possessing a number of properties presented in this statement. This result is based on Lemma 3.10 that states the existence of classical solutions of the Kolmogorov equation (1.2) in the form of (1.4), with $t \mapsto \phi_{t}$ described in Lemma 3.2 .

The rest of this paper has the following structure. In Sect.2, we settle the mathematical framework and formulate our assumptions concerning the branching kernel $b$. Then we introduce and describe tempered configurations by employing a continuous function $\psi: X \rightarrow(0,+\infty)$, that vanishes at infinite and is such that the aforementioned death probability satisfies $\delta(x) \geq 1-\psi(x)$. The key statement of this part is Proposition 2.1 according to which the set of all tempered configurations $\Gamma^{\psi}$ is a Polish space. This allows us to restrict ourselves to considering the states with the property $\mu\left(\Gamma^{\psi}\right)=1$. In fact, this restriction is a direct analog of the condition imposed in [10, see ibid, Definitions 3.1 and 3.2, page 223. Next, we discuss in detail the properties of the branching kernel. In Sect. 3, we prepare solving our main evolution equation (1.3) by defining $L$ as a closed linear operator with domain $\mathcal{D}(L)$ in the Banach space of bounded continuous functions $F: \Gamma^{\psi} \rightarrow \mathbb{R}$. As mentioned above, the key ingredient of this construction is solving the $\log$-Laplace lequation in the space of bounded continuous functions $\phi: X \rightarrow \mathbb{R}$, defined by the branching kernel, see Lemma 3.2. This step is typical in the theory of branching processes, cf. [3, Theorem 3.1]. With the help of this, we prove that the Kolmogorov equation (1.2) with the just mentioned closed Kolmogorov operator $L$ has a classical solution, see Lemma 3.10, in the form of (1.4) with $\phi_{t}$ solving the log-Laplace equation. Thereby and after additional preparations, we prove (Theorem 4.2) that (1.3) has a unique solution $t \mapsto \mu_{t}$, which is weakly continuous, i.e., $\mu_{t} \Rightarrow \mu_{s}$ as $t \rightarrow s$. In the subcritical case, we show that $\mu_{t} \Rightarrow \mu_{\infty}$ as $t \rightarrow+\infty$, where $\mu_{\infty}\left(\Gamma^{0}\right)=1$. At the very end, we make some concluding remarks on possible extensions of the results of this work.

\section{Preliminaries and Assumptions}

2.1. Notions and notations. By $\mathbb{1}_{A}$ we denote the indicator of a suitable set $A$. A Polish space is a separable topological space that can be metrized by a complete metric, see [7, Chapt. 8]. For a Polish space $E, \mathcal{B}(E)$ will stand for the corresponding Borel $\sigma$-field. By $C_{\mathrm{b}}(E), C_{\mathrm{cs}}(E), B_{\mathrm{b}}(E)$ we denote the sets of all continuous and bounded, continuous and compactly supported, measurable and bounded functions $f: E \rightarrow \mathbb{R}$, respectively. By $C_{\mathrm{b}}^{+}(E)$ we denote the set of positive elements of $C_{\mathrm{b}}(E)$. Finally, by $C_{0}^{+}(E)$ we denote the set of all $f \in C_{\mathrm{b}}^{+}(E)$ which satisfy: (a) $f(x)>0$ for all $X$; (b) for each $\varepsilon>0$, one finds a compact $\Lambda_{\varepsilon} \subset X$ such that $f(x)<\varepsilon$ whenever $x \in X \backslash \Lambda_{\varepsilon}$.

Let $\mathcal{F}$ be a family of functions $f: E \rightarrow \mathbb{R}$. By $\sigma \mathcal{F}$ we denote the smallest sub-field of $\mathcal{B}(E)$ such that each $f \in \mathcal{F}$ is $\sigma \mathcal{F} / \mathcal{B}(\mathbb{R})$-measurable. By $\mathcal{P}(E)$ we denote the set of all probability measures on $(E, \mathcal{B}(E))$; for suitable $f: E \rightarrow \mathbb{R}$ and $\mu \in \mathcal{P}(E)$, we write $\mu(f)=\int_{E} f d \mu$. The weak topology of $\mathcal{P}(E)$ is defined as the weakest one that makes continuous all the maps $\mu \mapsto \mu(f), f \in C_{\mathrm{b}}(E)$. With this topology $\mathcal{P}(E)$ is also a Polish space. By writing $\mu_{n} \Rightarrow \mu, n \rightarrow+\infty$, we mean that $\left\{\mu_{n}\right\}_{n \in \mathbb{N}}$ weakly converges to $\mu$. A family $\mathcal{F}$ of functions $f: E \rightarrow \mathbb{R}$ is called separating if $\mu_{1}(f)=\mu_{2}(f)$, holding for all $f \in \mathcal{F}$, implies $\mu_{1}=\mu_{2}$ for each pair $\mu_{1}, \mu_{2} \in \mathcal{P}(E)$. Furthermore, $\mathcal{F}$ is said to separate the points of $E$ if for each distinct $x, y \in E$, one finds $f \in \mathcal{F}$ with the property $f(x) \neq f(y)$. 
If $\mathcal{F}$ separates points and is closed with respect to multiplication, it is separating, see [12, Theorem 4.5, page 113]. A family $\mathcal{F}$ is called convergence determining if $\mu_{n}(f) \rightarrow \mu(f)$, holding for all $f \in \mathcal{F}$, implies $\mu_{n} \Rightarrow \mu$.

2.2. Tempered configurations. As mentioned above, we will deal with a locally compact Polish space $X$. Usually, branching processes are constructed in a more general setting, cf. [19], e.g., by taking as $X$ a Luzin space [2, 3]. Our choice of $X$ was done mostly for the following reason. In contrast to [2, 3], we study branching in infinite systems and thus deal with the space of infinite configurations. In Polish spaces without assuming their local compactness, the vague topology of spaces of infinite configurations is introduced with the help of continuous functions vanishing outside bounded sets, cf. [6, page 2], which assumes fixing some concrete metric of $X$. Thus, in order to be free in choosing such metrics, as well as to avoid possible complications of the topological aspects of our work, we restrict ourselves here to considering locally compact spaces. Note that the local compactness together with the separability of $X$ imply its $\sigma$-compactness, i.e, the existence of a nest of compact subsets $\left\{\Lambda_{k}\right\}_{k \in \mathbb{N}}$ that exhaust $X$. The existence of such nests and the corresponding compact Lyapunov functions could be taken as the basic topological assumption concerning $X$, as it was done in constructing quasi-regular Dirichlet forms [20], or cadlag processes by means of resolvent kernels [4].

Among all infinite configurations, one may distinguish those that have a priori prescribed properties. Here we do this by employing a function $\psi \in C_{\mathrm{b}}^{+}(X), \psi(x) \leq 1$, for which we set

$$
\Psi(\gamma)=\sum_{x \in \gamma} \psi(x)
$$

Then we define the set of tempered configurations as

$$
\Gamma^{\psi}=\{\gamma \in \Gamma: \Psi(\gamma)<\infty\}
$$

It is clear that

$$
\Gamma^{\psi^{\prime}} \supset \Gamma^{\psi}, \quad \text { whenever } \psi^{\prime} \leq \psi \text {. }
$$

By this observation we can vary $\Gamma^{\psi}$ from $\Gamma$ (by taking $\left.\psi \in C_{\mathrm{cs}}^{+}(X)\right)$ to $\Gamma_{0}:=\{\gamma \in \Gamma$ : $\gamma$ is finite , corresponding to $\psi \equiv 1$. If $\psi \in C_{0}^{+}(X)$, then $\Gamma^{\psi}$ is a proper subset of $\Gamma$ and supset of $\Gamma_{0}$. As an example. one can take $X=\mathbb{R}$ and $\psi(x)=\psi_{0} e^{-\alpha|x|}, \alpha>0$, $\psi_{0} \in(0,1]$. Then the configuration $\mathbb{N} \subset \mathbb{R}$ is in $\Gamma^{\psi}$, whereas $\{\log n: n \in \mathbb{N}\}$ is not if $\alpha \leq 1$. In a separate publication, we plan to study scales of such spaces $\Gamma^{\psi}$, including the inductive and projective limit topologies on $\cup_{\psi \in \Psi} \Gamma^{\psi}$ and $\cap_{\psi \in \Psi^{\prime}} \Gamma^{\psi}$, respectively, with suitable families $\Psi, \Psi^{\prime}$.

In the sequel, we employ one and the same $\psi \in C_{0}^{+}(X)$, separated away from zero, i.e., such that $\inf _{x \in \Lambda} \psi(x)>0$ for each compact $\Lambda \subset X$. Its choice will be done in the next subsection. For each $\gamma \in \Gamma^{\psi}$, the measure

$$
\nu_{\gamma}=\sum_{x \in \gamma} \psi(x) \delta_{x}
$$

is finite. Thus, one can equip $\Gamma^{\psi}$ with the topology defined as the weakest one that makes continuous all the maps

$$
\Gamma^{\psi} \ni \gamma \mapsto \sum_{x \in \gamma} g(x) \psi(x), \quad g \in C_{\mathrm{b}}(X) .
$$

Similarly as in Proposition 2.7 and Corollary 2.8 of [17], we prove the following.

Proposition 2.1. With the topology defined in 2.4, $\Gamma^{\psi}$ is a Polish space, continuously embedded in $\Gamma$. Thus, $\mathcal{B}\left(\Gamma^{\psi}\right)=\left\{A \in \mathcal{B}(\Gamma): A \subset \Gamma^{\psi}\right\}$. 
Proof. First we note that the set of measures $\left\{\nu_{\gamma}: \gamma \in \Gamma^{\psi}\right\}$ is a subset of the space $\mathcal{M}$ of all finite positive Borel measures on $X$, which is a Polish space with the weak topology. Let us prove that $\left\{\nu_{\gamma}: \gamma \in \Gamma^{\psi}\right\}$ is a closed subset of $\mathcal{M}$. To this end, we take a sequence $\left\{\gamma_{n}\right\}_{n \in \mathbb{N}} \subset \Gamma^{\psi}$ such that $\left\{\nu_{\gamma_{n}}\right\}_{n \in \mathbb{N}}$ is a Cauchy sequence in a metric of $\mathcal{M}$ that makes this space complete. Let $\nu \in \mathcal{M}$ be its limit, and hence

$$
\sum_{x \in \gamma_{n}} g(x) \psi(x) \rightarrow \nu(g), \quad n \rightarrow+\infty
$$

holding for all $g \in C_{\mathrm{b}}(X)$, in particular for $g \in C_{\mathrm{cs}}(X)$. Since $\psi$ is separated away from zero, each $h \in C_{\mathrm{cs}}(X)$ can be written in the form $h(x)=g(x) \psi(x)$ with $g \in C_{\mathrm{cs}}(X)$. It is known, see, e.g., [23, page 397], that there exists a countable family $\left\{h_{k}\right\}_{k \in \mathbb{N}} \subset C_{\text {cs }}(X)$ such that

$$
\bar{v}\left(\gamma, \gamma^{\prime}\right):=\sum_{k \in \mathbb{N}} 2^{-k} \frac{\left|\sum_{x \in \gamma} h_{k}(x)-\sum_{x \in \gamma^{\prime}} h_{k}(x)\right|}{1+\left|\sum_{x \in \gamma} h_{k}(x)-\sum_{x \in \gamma^{\prime}} h_{k}(x)\right|}
$$

is a complete metric of $\Gamma$. Then the convergence as in (2.5) yields that the sequence $\left\{\gamma_{n}\right\}_{n \in \mathbb{N}}$ converges to some $\gamma \in \Gamma$ in the vague topology of $\Gamma$. To prove that this $\gamma$ lies in $\Gamma^{\psi}$, we take an ascending sequence of compact $\Lambda_{m} \subset X$, i.e., such that each $\Lambda_{m}$ lies in the interior of $\Lambda_{m+1}$ and each $x \in X$ is contained in some $\Lambda_{m}$. Then we take $g^{(m)} \in C_{\mathrm{cs}}(X)$ such that $g^{(m)}(x)=1$ for $x \in \Lambda_{m}$, and $g^{(m)}(x)=0$ for $x \in X \backslash \Lambda_{m+1}$, which is possible by Urysohn's lemma. Then

$$
\sum_{x \in \gamma} g^{(m)}(x) \psi(x)=\nu\left(g^{(m)}\right) \leq \nu(X) .
$$

Now we pass here to the limit $m \rightarrow+\infty$ and obtain (by the Beppo Levi theorem) that $\Psi(\gamma) \leq \nu(X)$, which yields, $\gamma \in \Gamma^{\psi}$. Thus, $\left\{\nu_{\gamma}: \gamma \in \Gamma^{\psi}\right\}$ is closed in $\mathcal{M}$, and thereby is also Polish, see [7, Proposition 8.1.2, page 240]. This yields the first half of the statement. The stated continuity of the embedding $\Gamma^{\psi} \hookrightarrow \Gamma$ is immediate. Then the conclusion concerning the $\sigma$-fields follows by Kuratowski's theorem, see [21, Theorem 3.9, page 21].

Remark 2.2. The continuity of the embedding $\Gamma^{\psi} \hookrightarrow \Gamma$ allows one to establish the following fact:

$$
\mathcal{P}\left(\Gamma^{\psi}\right)=\left\{\mu \in \mathcal{P}(\Gamma): \mu\left(\Gamma^{\psi}\right)=1\right\} .
$$

That is, each $\mu \in \mathcal{P}(\Gamma)$ possessing the property $\mu\left(\Gamma^{\psi}\right)=1$ can be redefined as a probability measure on $\Gamma^{\psi}$. Therefore, by restricting ourselves to tempered configurations - members of $\Gamma^{\psi}$ - we exclude from our consideration all those $\mu \in \mathcal{P}(\Gamma)$ that fail to satisfy the mentioned support condition. At the same time, the map $\Gamma^{\psi} \ni \gamma \mapsto \nu_{\gamma} \in \mathcal{M}$, see (2.3), defines a natural embedding $\Gamma^{\psi} \hookrightarrow \mathcal{M}$ and thus pushes each $\mu \in \mathcal{P}\left(\Gamma^{\psi}\right)$ to a probability measure on $\mathcal{M}$. For obvious reasons, below we do not distinguish between the elements of $\mathcal{P}\left(\Gamma^{\psi}\right)$ and the corresponding push-forward measures on $\mathcal{M}$.

Let $E$ be a Polish space. Following [12, page 111], we say that a sequence $\left\{h_{n}\right\}_{n \in \mathbb{N}} \subset$ $B_{\mathrm{b}}(E)$ converges to a certain $h \in B_{\mathrm{b}}(E)$ boundedly and pointwise if: (a) $\sup _{n}\left\|h_{n}\right\|<\infty$; (b) $h_{n}(x) \rightarrow h(x)$ for each $x \in E$. In this case, we write $h_{n} \stackrel{b p}{\rightarrow} h$. A subset, $\mathcal{H} \subset B_{\mathrm{b}}(E)$, is said to be $b p$-closed, if $\left\{h_{n}\right\} \subset \mathcal{H}$ and $h_{n} \stackrel{b p}{\rightarrow} h$ imply $h \in \mathcal{H}$. The $b p$-closure of $\mathcal{H} \subset B_{\mathrm{b}}(E)$ is the smallest $b p$-closed subset of $B_{\mathrm{b}}(E)$ that contains $\mathcal{H}$. An $\mathcal{H}^{\prime}$ is $b p$-dense in $\mathcal{H}$, if the latter is the smallest $b p$-closed set that contains $\mathcal{H}^{\prime}$. The following is known, see [12, Proposition 4.2, page 111] and/or [8, Lemmas 3.2.1, 3.2.3, pages 41, 42].

Proposition 2.3. For each Polish space E, there exists a countable family $\mathcal{H} \subset C_{\mathrm{b}}^{+}(E)$ that has the following properties: (a) the linear span of $\mathcal{H}$ is bp-dense in $B_{\mathrm{b}}(E)$; (b) $\mathcal{B}(E)=\sigma \mathcal{H} ;(c)$ it contains the unit function $u(x) \equiv 1$ and is closed with respect to addition; (d) it is separating; (e) it is convergence determining. 
Let $\mathcal{V}=\left\{v_{l}\right\}_{l \in \mathbb{N}} \subset C_{\mathrm{b}}^{+}(X)$ be a family of functions with the property as in Proposition 2.3. We may and will assume that each $v_{l} \in \mathcal{V}$ satisfies $\inf _{X} v_{l}(\hat{x}) \geq c_{0, l}>0$ for an appropriate $c_{0, l}$, cf. [8, Remark 3.2.3, page 42]. Indeed, if this is not the case, instead of $v_{l}$ one can take $\tilde{v}_{l}:=v_{l}+c_{0, l}$. Then the family $\left\{\tilde{v}_{l}\right\}_{l \in \mathbb{N}}$ has all the properties we need. For $\gamma \in \Gamma^{\psi}$, we have, cf. (2.3),$\nu_{\gamma}\left(v_{l}\right)=\sum_{x \in \gamma} v_{l}(x) \psi(x)$. The topology mentioned in Proposition 2.1 is metrizable with the metric

$$
v_{*}\left(\gamma, \gamma^{\prime}\right)=\sum_{l=0}^{\infty} \frac{2^{-l}\left|\nu_{\gamma}\left(v_{l}\right)-\nu_{\gamma^{\prime}}\left(v_{l}\right)\right|}{1+\left|\nu_{\gamma}\left(v_{l}\right)-\nu_{\gamma^{\prime}}\left(v_{l}\right)\right|} .
$$

For $\mu \in \mathcal{P}\left(\Gamma^{\psi}\right)$, its Laplace transform is defined by the expression

$$
\begin{gathered}
\mathfrak{L}_{\mu}(g)=\mu\left(G^{g}\right), \quad g \in C_{\mathrm{b}}^{+}(X) \\
G^{g}(\gamma):=\exp \left(-\nu_{\gamma}(g)\right)=\exp \left(-\sum_{x \in \gamma} g(x) \psi(x)\right) .
\end{gathered}
$$

It is known, see [8, Lemma 3.2.5 and Theorem 3.2.6, page 43], that the Laplace transforms of probability measures on $\mathcal{M}$ have a number of useful properties which we are going to exploit. In view of the embedding mentioned in the second part of Remark 2.2, we can attribute these properties also to the transform defined in (2.8).

Proposition 2.4. Let $\mathcal{V}$ be the family of functions used in (2.7). Then:

(i) $\mathcal{B}\left(\Gamma^{\psi}\right)=\sigma\left\{G^{v}: v \in \mathcal{V}\right\}$;

(ii) $B_{\mathrm{b}}\left(\Gamma^{\psi}\right)$ is the bp-closure of the linear span of $\left\{G^{v}: v \in \mathcal{V}\right\}$;

(iii) $\left\{G^{v}: v \in \mathcal{V}\right\}$ is separating;

(iv) $\left\{G^{v}: v \in \mathcal{V}\right\}$ is convergence determining.

The proof of claim (iv) is essentially based on the concrete choice of the metric (2.7), by which one shows that the family $\left\{G^{v}: v \in \mathcal{V}\right\}$ is strongly separating, cf. [12, page 113].

In the sequel, we will use the functions

$$
\phi(x)=1-\theta(x)=\exp (-g(x) \psi(x)),
$$

with $g \in C_{\mathrm{b}}^{+}(X)$, that includes also the choice $g \in \mathcal{V}$.

2.3. The branching kernel. We assume that, for each $x \in X, b_{x} \in \mathcal{P}(\Gamma)$ is such that $b_{x}\left(\Gamma_{0}\right)=1$, i.e., each cloud of offsprings is finite. Recall that $\Gamma_{0} \in \mathcal{B}(\Gamma)$ is the set of all finite configurations. For $n \in \mathbb{N}_{0}$, we set $\Gamma^{n}=\left\{\xi \in \Gamma_{0}:|\xi|=n\right\}$. Then $b_{x}\left(\Gamma^{n}\right)$ is the probability of producing $n$ offsprings by the particle located at $x$. Note that $\delta(x):=b_{x}\left(\Gamma^{0}\right)$ is just the death probability, and

$$
n(x):=\int_{\Gamma_{0}}|\xi| b_{x}(d \xi)=\sum_{n=1}^{\infty} n b_{x}\left(\Gamma_{n}\right)=\beta_{x}^{(1)}(X)
$$

is the expected number of offsprings of the particle located at $x$. Here $\beta_{x}^{(1)}$ is the first correlation measure of $b_{x}$, see, e.g., [18] for a rigorous definition. Here we use its property

$$
\int_{\Gamma}\left(\sum_{y \in \xi} h(y)\right) b_{x}(d \xi)=\int_{X} h(y) \beta_{x}^{(1)}(d y) .
$$

For $\phi$ as in (2.9), we define

$$
(\Phi \phi)(x)=\int_{\Gamma}\left(\prod_{y \in \xi} \phi(y)\right) b_{x}(d \xi),
$$


with the convention that $\prod_{x \in \varnothing} \phi(x)=1$. Clearly, $0 \leq(\Phi \phi)(x) \leq 1$ for each $x \in X$. Recall that we use $\psi$ in (2.1) in defining tempered configurations.

Assumption 2.5. The probability kernel $b$ is subject to the following conditions:

(i) $\Phi \phi \in C_{\mathrm{b}}(X)$ for each $\phi$ as in (2.9);

(ii) $\sup _{x \in X} n(x)=: n_{*}<\infty$;

(iii) the death probability $\delta$ satisfies $\delta(x) \geq 1-\psi(x) \geq \delta_{*}>0$, holding for all $x \in X$;

(iv) there exists $m>0$ such that, for all $x \in X$, the following holds

$$
\int_{X} \psi(y) \beta_{x}^{(1)}(d y) \leq n(x) m \psi(x) .
$$

By (2.9), (2.11), (2.13) and Jensen's inequality one gets

$$
\begin{gathered}
-\log (\Phi \phi)(x) \leq \int_{\Gamma_{0}}\left(-\log \prod_{y \in \xi} \phi(y)\right) b_{x}(d \xi) \\
=\int_{X} g(y) \psi(y) \beta_{x}^{(1)}(d y) \leq\left(\sup _{x \in X} g(x)\right) n(x) m \psi(x) .
\end{gathered}
$$

Note that by (2.8), (2.12) and our choice $\phi=e^{-g \psi}$ it follows that

$$
(\Phi \phi)(x)=\int_{\Gamma_{0}} G^{g}(\xi) b_{x}(d \xi)=\mathfrak{L}_{b_{x}}(g) .
$$

Then assumption (i) can be reformulated as the continuity of the map $X \ni x \mapsto \mathfrak{L}_{b_{x}}(g) \in$ $\mathbb{R}$, holding for all $g \in C_{\mathrm{b}}^{+}(X)$. The remaining assumptions are being made to control the production of new particles, of which (ii) and (iii) are related to the properties of $b_{x}\left(\Gamma^{n}\right)$, $n \in \mathbb{N}_{0}$, see (2.10). In general, (ii) and (iii) may be quite independent as the choice of $\delta(x)$ leaves enough possibilities to modify $n(x)$. However, in some cases, $\delta(x)$ and $n(x)$ can be expressed through each other. For instance, if $b_{x}$ is a Poisson measure - which is completely determined by its first correlation measure that appears in (2.10), see, e.g., [8, page 45] - then $\delta(x)=e^{-n(x)}$. In this case, (ii) follows by (iii) with $n_{*}=-\log \delta_{*}$. The role of (iv) is to control the dispersal of offsprings, and thus the nonlocality of the process. To illustrate its role, we take $X=\mathbb{R}$ and

$$
\bar{\beta}_{x}(d y):=\beta_{x}^{(1)}(d y) / n(x)=\frac{1}{2 r} \mathbb{1}_{[x-r, x+r]}(y) d y, \quad r>0 .
$$

Then $\psi(y)=\left(1-\delta_{*}\right) e^{-\alpha|y|}$ satisfies

$$
\int_{X} \psi(y) \bar{\beta}_{x}(d y) \leq\left(\frac{e^{\alpha r}-e^{-\alpha r}}{2 \alpha r}\right) \psi(x)
$$

which yields (2.13) with $m=\sinh (\alpha r) / \alpha r>1$. Note that this $m$ can be made arbitrarily close to one by taking small enough either $r$ or $\alpha$. The former corresponds to a short dispersal, whereas by choosing small $\alpha$ one makes $\Gamma^{\psi}$ - and hence $\mathcal{P}\left(\Gamma^{\psi}\right)$ - smaller, cf. (2.2) and (2.6).

\section{The Kolmogorov Equation}

Our aim in this section is to solve (1.2) and to prepare solving (1.3). 
3.1. Solving the log-Laplace equation. As is typical for the theory of measure-valued branching processes, see [2, 3] and [8, Chapt. 4], the main point of their constructing is solving a nonlinear evolution equation, often called 'log-Laplace equation', see [8, pp. 60, 61]. We approach this by defining

$$
C_{\psi}(X)=\left\{\phi \in C_{\mathrm{b}}(X): \forall x \in X \quad 0<c_{\phi} \psi(x) \leq 1-\phi(x)=: \theta(x) \leq 1-\delta(x)\right\},
$$

i.e., each $\theta=1-\phi$ has its own lower bound, whereas the upper bound is one and the same for all such functions. Notably, by item (iii) of Assumption 2.5 it follows that each $\phi \in C_{\psi}(X)$ satisfies

$$
\phi(x) \geq 1-\psi(x) \geq \delta_{*} .
$$

Let us prove that $(\Phi \phi)(x) \geq \delta(x)$, holding for each $\phi \in C_{\psi}(X)$. Since $\phi(y) \geq 0$, by (2.12) we have

$$
(\Phi \phi)(x) \geq \int_{\Gamma^{0}} b_{x}(d \xi)=b_{x}\left(\Gamma^{0}\right)=\delta(x) \geq 1-\psi(x) \geq \delta_{*},
$$

see item (iii) of Assumption 2.5. Moreover, by (2.9) and (3.2) it follows that

$$
g(x) \leq-\frac{1}{\psi(x)} \log (1-\psi(x))=\sum_{n=1}^{\infty} \frac{[\psi(x)]^{n-1}}{n} \leq-\frac{\log \left(1-\delta_{*}\right)}{1-\delta_{*}}=: g_{*} .
$$

Both (3.2) and (3.4) holding for all $x \in X$.

Now for $T>0$, by $\mathcal{C}^{T}$ we denote the Banach space of continuous maps $[0, T] \ni t \mapsto$ $\varphi_{t} \in C_{\mathrm{b}}(X)$, equipped with the norm

$$
\|\varphi\|_{T}=\sup _{t \in[0, T]} \sup _{x \in X}\left|\varphi_{t}(x)\right| .
$$

We also set

$$
\mathcal{C}_{\psi}^{T}=\left\{\varphi \in \mathcal{C}^{T}: \varphi_{t} \in C_{\psi}(X), t \in[0, T]\right\}
$$

and

$$
\mathcal{C}_{\psi}^{T}(\phi)=\left\{\varphi \in \mathcal{C}_{\psi}^{T}: \varphi_{0}=\phi, \quad \varphi_{t}(x) \leq 1-c_{\phi} e^{-t} \psi(x)\right\}, \quad \phi \in C_{\psi}(X),
$$

where $c_{\phi}$ is the same as in (3.1) for this $\phi$. Clearly, $\mathcal{C}_{\psi}^{T}(\phi)$ is a closed subset of $\mathcal{C}_{\psi}^{T}$. Indeed, let $\left\{\varphi_{n}\right\}_{n \in \mathbb{N}} \subset \mathcal{C}_{\psi}^{T}(\phi)$ be $\|\cdot\|_{T}$-convergent to a certain $\varphi \in \mathcal{C}^{T}$. Then $\varphi_{0}=\phi$ and $\varphi_{t}(x) \leq 1-c_{\phi} e^{-t} \psi(x)$ since $\left(\varphi_{n}\right)_{t}(x) \rightarrow \varphi_{t}(x)$ as $n \rightarrow+\infty$, holding for all $t \in[0, T]$ and $x \in X$. Now we define

$$
(K \varphi)_{t}(x)=\varphi_{0}(x) e^{-t}+\int_{0}^{t} e^{-(t-s)}\left(\Phi \varphi_{s}\right)(x) d s .
$$

Proposition 3.1. Let $n_{*}$ introduced in Assumption 2.5 and T satisfy $n_{*}\left(1-e^{-T}\right)<1$. Then for each $\phi \in C_{\psi}(X)$, the map $K$ has a unique fixed point $\varphi \in \mathcal{C}_{\psi}^{T}(\phi)$.

Proof. We begin by showing that $K: \mathcal{C}_{\psi}^{T}(\phi) \rightarrow \mathcal{C}_{\psi}^{T}(\phi)$ for each $T>0$. Clearly, $x \mapsto$ $(K \varphi)_{t}(x)$ is continuous and $(K \varphi)_{0}=\phi$ whenever $\varphi \in \mathcal{C}_{\psi}^{T}(\phi)$. The continuity of $t \mapsto \Phi \varphi_{t}$ follows by the estimate, see (2.12),

$$
\begin{aligned}
& \left|\left(\Phi \varphi_{s}\right)(x)-\left(\Phi \varphi_{u}\right)(x)\right| \leq \int_{\Gamma_{0}}\left|\prod_{y \in \xi} \varphi_{s}(y)-\prod_{y \in \xi} \varphi_{u}(y)\right| b_{x}(d \xi) \\
& \quad \leq \sup _{y \in X}\left|\varphi_{s}(y)-\varphi_{u}(y)\right| \int_{\Gamma_{0}}|\xi| b_{x}(d \xi) \leq n_{*} \sup _{y \in X}\left|\varphi_{s}(y)-\varphi_{u}(y)\right| .
\end{aligned}
$$

This also yields the continuity of $t \mapsto(K \varphi)_{t}$. In obtaining (3.8) we have used the following evident estimate

$$
\left|a_{1} a_{2} \cdots a_{n}-b_{1} b_{2} \cdots b_{n}\right| \leq n \max _{i}\left|a_{i}-b_{i}\right|, \quad a_{i}, b_{i} \in[0,1] .
$$


Furthermore,

$$
0<(K \varphi)_{t}(x) \leq \phi(x) e^{-t}+\left(1-e^{-t}\right)=1-(1-\phi(x)) e^{-t} \leq 1
$$

which yields

$$
1-(K \varphi)_{t}(x) \geq e^{-t} \theta(x) \geq e^{-t} c_{\phi} \psi(x)=: c_{\phi}(t) \psi(x),
$$

and hence the validity of the upper estimate assumed in (3.6). Similarly as in (3.3) we have

$$
\left(\Phi \varphi_{s}\right)(x) \geq b_{x}\left(\Gamma^{0}\right)=\delta(x) \geq 1-\psi(x),
$$

where we used also item (iii) of Assumptions 2.5. By means of this estimate applied in (3.7) we then get

$$
\begin{gathered}
(K \varphi)_{t}(x) \geq \phi(x) e^{-t}+\left(1-e^{-t}\right) \delta(x) \\
\geq(1-\psi(x))+e^{-t}(\phi(x)-\delta(x)) \geq 1-\psi(x),
\end{gathered}
$$

as $\phi \in C_{\psi}(X)$. Thus, $K: \mathcal{C}_{\psi}^{T}(\phi) \rightarrow \mathcal{C}_{\psi}^{T}(\phi)$. Let us show that it is a contraction. To this end, similarly as in (3.8) we obtain, see also (3.5),

$$
\|K \varphi-K \tilde{\varphi}\|_{T} \leq n_{*}\left(1-e^{-T}\right)\|\varphi-\tilde{\varphi}\|_{T}, \quad \varphi, \tilde{\varphi} \in \mathcal{C}_{\psi}^{T}(\phi) .
$$

Now the proof follows by Banach's contraction principle.

Next we consider the following nonlinear equation

$$
\frac{\partial}{\partial t} \phi_{t}(x)=-\phi_{t}(x)+\left(\Phi \phi_{t}\right)(x), \quad \phi_{0}=\phi .
$$

It is a nonlocal analog of the log-Laplace equation - a standard object in the theory of branching processes, see, e.g., [8, page 61]. By a classical solution of (3.10) we will understand a map $\mathbb{R}_{+} \ni t \mapsto \phi_{t} \in C_{\mathrm{b}}(X)$ which is everywhere continuously differentiable and satisfies both equalities mentioned therein.

Lemma 3.2. For each $\phi \in C_{\psi}(X)$, (3.10) has a unique solution $t \mapsto \phi_{t} \in C_{\psi}(X)$ which satisfies

$$
c_{\phi}(t) \psi(x) \leq 1-\phi_{t}(x)=: \theta_{t}(x) \leq \psi(x),
$$

with $c_{\phi}(t)$ defined in (3.9). For $n_{*}<1$, this solution tends to $\phi_{\infty}(x) \equiv 1$ as $t \rightarrow+\infty$ in the norm of $C_{\mathrm{b}}(X)$.

Proof. We begin by fixing $T>0$ such that the contraction condition $n_{*}\left(1-e^{-T}\right)<1$ is satisfied. Then integrating in (3.10) we arrive at the following integral equation

$$
\phi_{t}(x)=\phi(x) e^{-t}+\int_{0}^{t} e^{-(t-s)}\left(\Phi \phi_{s}\right)(x) d s,
$$

the set of solutions of which on $[0, T]$ coincides with the set of fixed points of $K: \mathcal{C}^{T}(\phi) \rightarrow$ $\mathcal{C}^{T}(\phi)$ established in Proposition 3.1. The continuous differentiability of $t \mapsto \phi_{t} \in C_{\mathrm{b}}(X)$ follows by continuity $s \mapsto \Phi \psi_{s}$, which in turn follows by (3.8). Thus, each solution of (3.12) solves also (3.10), which yields the existence of the solution in question on the time interval $[0, T]$. For $n_{*} \leq 1$, the contraction condition is satisfied for any $T>0$; hence, the aforementioned solution is global in time. For $n_{*}>1$, we proceed as follows. For 
$t_{1}+t_{2} \leq T$, we rewrite (3.12) as follows

$$
\begin{aligned}
\phi_{t_{1}+t_{2}}(x)= & e^{-t_{2}}\left(\phi(x) e^{-t_{1}}+\int_{0}^{t_{1}} e^{-\left(t_{1}-s\right)}\left(\Phi \phi_{s}\right)(x) d s\right) \\
& +\int_{t_{1}}^{t_{1}+t_{2}} e^{-\left(t_{2}+t_{1}-s\right)}\left(\Phi \phi_{s}\right)(x) d s \\
= & \phi_{t_{1}}(x) e^{-t_{2}}+\int_{0}^{t_{2}} e^{-\left(t_{2}-s\right)}\left(\Phi \phi_{t_{1}+s}\right)(x) d s .
\end{aligned}
$$

Since the contraction condition is independent of the initial condition in (3.10), by (3.13) one can continue the solution obtained above to any $t>0$. Indeed, let $\phi_{t}$ be the solution on $[0, T]$. Let also $\phi_{t}^{1} \in \mathcal{C}_{\psi}^{T}\left(\phi^{1}\right)$ be the solution of (3.10) on the same $[0, T]$ with the initial condition $\phi_{t}^{1}:=\phi_{T / 2}$. By the uniqueness established in Lemma 3.2 it follows that these two solutions satisfy $\phi_{t+T / 2}=\phi_{t}^{1}$ for $t \in[0, T / 2]$. Hence, the function $\phi_{t} \mathbb{1}_{[0, T / 2]}(t)+$ $\phi_{t-T / 2}^{1} \mathbb{1}_{[T / 2,3 T / 2]}(t)=\phi_{t} \mathbb{1}_{[0, T]}(t)+\phi_{t-T}^{1} \mathbb{1}_{[T, 3 T / 2]}(t)$ is the unique solution of (3.12) (hence of (3.10) $)$ on $[0,3 T / 2]$. The further continuation goes in analogous way.

For $n_{*}<1$, we define $\vartheta_{s}=e^{s}\left\|1-\phi_{s}\right\|=e^{s} \sup _{x \in X}\left(1-\phi_{s}(x)\right)$. By (3.12) we then get

$$
\vartheta_{t} \leq \vartheta_{0}+n_{*} \int_{0}^{t} \vartheta_{s} d s
$$

which by Grönwall's inequality yields,

$$
\left\|1-\phi_{t}\right\| \leq\|1-\phi\| e^{-\left(1-n_{*}\right) t}
$$

and thereby the convergence in question. Note that $\phi_{\infty}$ does not belong to $C_{\psi}(X)$ as it fails to obey the upper bound $\phi(x) \leq 1-c_{\phi} \psi(x)$ with $c_{\phi}>0$, see (3.1). However, it belongs to the closure of this set, and is a stationary solution of (3.10).

Remark 3.3. By (3.13) it follows that the solution of (3.10) - which is a nonlinear Cauchy problem in the Banach space $C_{\mathrm{b}}(X)$ - is given by a continuous semigroup of nonlinear operators, say $\left\{\rho_{t}\right\}_{t \geq 0}$, in the form $\phi_{t}=\rho_{t}\left(\phi_{0}\right), \phi_{t} \in C_{\psi}(X)$. If one writes $\phi_{t} \in C_{\psi}(X)$ in the form $\phi_{t}(x)=\exp \left(-g_{t}(x) \psi((x))\right.$, see (2.9), then the map $g \mapsto g_{t}$ also has the flow property. It defines a continuous semigroup of nonlinear operators $\left\{r_{t}\right\}_{t \geq 0}$ such that $g_{t}=r_{t}\left(g_{0}\right)$. It is known as the log-Laplace semigroup, see [8, page 60].

We conclude this subsection by establishing the following useful properties of the solution $\phi_{t}$.

Lemma 3.4. Let $\phi_{t}=1-\theta_{t}-e^{-g_{t} \psi}$ be the solution as in Lemma 3.2. Then, for each $t \geq 0, u>0$ and all $x \in X$, the following holds

$$
\begin{array}{ll}
\text { (a) } & \left|\phi_{t+u}(x)-\phi_{t}(x)\right|=\left|\theta_{t+u}(x)-\theta_{t}(x)\right| \leq 2 u \psi(x), \\
\text { (b) } & \left|g_{t+u}(x)-g_{t}(x)\right| \leq 2 u / \delta_{*}, \\
\text { (c) } & \left|\left(\Phi \phi_{t+u}\right)(x)-\left(\Phi \phi_{t}\right)(x)\right| \leq 2 u n_{*} m \psi(x) .
\end{array}
$$

Proof. By (3.10) we have

$$
\begin{aligned}
& \left|\phi_{t+u}(x)-\phi_{t}(x)\right| \leq \int_{0}^{u}\left|\phi_{t+s}(x)-\left(\Phi \phi_{t+s}\right)(x)\right| d s \\
& =\int_{0}^{u}\left|\theta_{t+s}(x)-\left(1-\left(\Phi \phi_{t+s}\right)(x)\right)\right| d s \leq 2 \psi(x) u,
\end{aligned}
$$

where we have used (3.11) and (3.3). To prove (b), we denote

$$
h^{+}(x)=\max \left\{g_{t+u}(x) \psi(x) ; g_{t}(x) \psi(x)\right\}, \quad h^{-}(x)=\min \left\{g_{t+u}(x) \psi(x) ; g_{t}(x) \psi(x)\right\} .
$$


Then, cf. (2.9),

$$
\begin{aligned}
\mid \phi_{t+u}(x) & -\phi_{t}(x) \mid=e^{-h^{+}(x)}\left[e^{h^{+}(x)-h^{-}(x)}-1\right] \\
& \geq e^{-h^{+}(x)}\left|g_{t+u}(x)-g_{t}(x)\right| \psi(x) \\
& \geq \min \left\{\phi_{t+u}(x) ; \phi_{t}(x)\right\}\left|g_{t+u}(x)-g_{t}(x)\right| \psi(x),
\end{aligned}
$$

which yields case (b) of (3.14) by (3.15) and (3.2). Next, similarly as in (3.8) we get

$$
\begin{aligned}
\mid\left(\Phi \phi_{t+u}\right)(x) & -\left(\Phi \phi_{t}\right)(x) \mid \leq \int_{\Gamma_{0}}\left(\sum_{y \in \xi}\left|\phi_{t+u}(y)-\phi_{t}(y)\right|\right) b_{x}(d \xi) \\
& \leq 2 u \int_{X} \psi(y) \beta_{x}^{(1)}(d y) \leq 2 u n_{*} m \psi(x),
\end{aligned}
$$

where we used (3.15) and (2.13), see also item (i) of Assumption 2.5.

3.2. Basic estimates. In defining $L$, we employ a number of estimates which we derive now. For $\phi=e^{-g \psi} \in C_{\psi}(X)$ we set, see (2.9),

$$
F^{\phi}(\gamma)=\prod_{x \in \gamma} \phi(x)=\exp \left(-\sum_{x \in \gamma} g(x) \psi(x)\right)=G^{g}(\gamma),
$$

where $G^{g}(\gamma)$ is as in (2.8).

Proposition 3.5. Let $F^{\phi}$ be as in (3.16) with $\phi \in C_{\psi}(X)$, see (3.1). Then, for each $\gamma \in \Gamma^{\psi}$, the following holds

$$
\left|L F^{\phi}(\gamma)\right| \leq \frac{2}{e \delta_{*} c_{\phi}},
$$

where $c_{\phi}$ defines the lower bound in (3.1). By (3.17) it then follows that $L F^{\phi} \in C_{\mathrm{b}}\left(\Gamma^{\psi}\right)$. Proof. By (1.1), and then by (3.2), (3.3) and (3.1), we have

$$
\begin{gathered}
\left|L F^{\phi}(\gamma)\right| \leq \sum_{x \in \gamma} F^{\phi}(\gamma \backslash x)|(\Phi \phi)(x)-\phi(x)| \\
\leq\left(F^{\phi}(\gamma) / \delta_{*}\right) \sum_{x \in \gamma}(|1-(\Phi \phi)(x)|+|1-\phi(x)|) \\
\leq 2 \Psi(\gamma) F^{\phi}(\gamma) / \delta_{*} \leq 2 F^{\phi}(\gamma) e^{c_{\phi} \Psi(\gamma)} /\left(e \delta_{*} c_{\phi}\right) \leq 2 /\left(e \delta_{*} c_{\phi}\right),
\end{gathered}
$$

where $\Psi$ is as in (2.1). To get the latter two estimates in (3.18), we proceeded as follows. The first one was obtained with the help of the estimate $\alpha \leq e^{\alpha-1}, \alpha \geq 0$. Afterwards, we estimated

$$
F^{\phi}(\gamma) \exp \left(c_{\phi} \Psi(\gamma)\right)=\prod_{x \in \gamma}(1-\theta(x)) e^{c_{\phi} \psi(x)} \leq \prod_{x \in \gamma}\left(1-c_{\phi} \psi(x)\right) e^{c_{\phi} \psi(x)} \leq 1,
$$

see (3.1), which was used in the final step. The continuity of the map $\gamma \mapsto L F^{\phi}(\gamma)$ follows by the very definition of the topology of $\Gamma^{\psi}$.

As in (3.1) we do not restrict the lower bounds, the right-hand side of (3.17) can be arbitrarily large for small enough $c_{\phi}$.

Corollary 3.6. For a given $\phi \in C_{\psi}(X)$, let $\phi_{t}$ be the solution mentioned in Lemma 3.2. Then for each $\gamma \in \Gamma^{\psi}$, the map $t \mapsto F^{\phi_{t}}(\gamma)$ is continuously differentiable on $\mathbb{R}_{+}$and the following holds

$$
\frac{d}{d t} F^{\phi_{t}}(\gamma)=L F^{\phi_{t}}(\gamma)
$$


Proof. Recall that $\phi_{t}(x)=\exp \left(-g_{t}(x) \psi(x)\right)$. Then the continuous differentiability of $t \mapsto g_{t}(x)$ follows by the analogous property of $t \mapsto \phi_{t}(x)$, see Lemma 3.2. Indeed, by case (b) of (3.14) it follows that the derivative of the former map is bounded uniformly in $x$, which yields that the map $t \mapsto \sum_{x \in \gamma} g_{t}(x) \psi(x)$ is continuously differentiable for each $\gamma \in \Gamma^{\psi}$. This implies the same property for the map $t \mapsto F^{\phi_{t}}(\gamma)$, as well as the boundedness of the map $\gamma \mapsto(d / d t) F^{\phi_{t}}(\gamma)$. The latter is proved analogously as in (3.18). Finally, the equality in (3.20) follows by the fact that $\phi_{t}$ solves (3.10).

Our next step is obtaining a number of useful estimates characterizing the map $t \mapsto$ $F^{\phi_{t}}(\gamma)$.

Lemma 3.7. For a given $\phi \in C_{\psi}(X)$, let $\phi_{t}$ be the solution of (3.10), see Lemma 3.2. Then, for each $t \geq 0, u>0$ and $\gamma \in \Gamma^{\psi}$, the following holds

$$
\left|F^{\phi_{t+u}}(\gamma)-F^{\phi_{t}}(\gamma)\right| \leq \frac{2 u e^{t+u}}{e \delta_{*} c_{\phi}} .
$$

Proof. We fix $t$ and $u$ and define

$$
\begin{gathered}
H_{s}(\gamma)=\sum_{x \in \gamma} g_{s}(x) \psi(x), \quad H^{+}(\gamma)=\max \left\{H_{t+u}(\gamma) ; H_{t}(\gamma)\right\} \\
H^{-}(\gamma)=\min \left\{H_{t+u}(\gamma) ; H_{t}(\gamma)\right\}
\end{gathered}
$$

Then

$$
\begin{aligned}
& \left|F^{\phi_{t+u}}(\gamma)-F^{\phi_{t}}(\gamma)\right|=e^{-H^{+}(\gamma)}\left[e^{H^{+}(\gamma)-H^{-}(\gamma)}-1\right] \\
& \leq \max \left\{F^{\phi_{t+u}}(\gamma) ; F^{\phi_{t}}(\gamma)\right\} \sum_{x \in \gamma}\left|g_{t+u}(x)-g_{t}(x)\right| \psi(x) \\
& \leq \frac{2 u}{\delta_{*}} \Psi(\gamma) \prod_{x \in \gamma}\left(1-c_{\phi}(t+u) \psi(x)\right) \\
& \leq \frac{2 u}{e \delta_{*} c_{\phi}(t+u)} \prod_{x \in \gamma}\left(1-c_{\phi}(t+u) \psi(x)\right) e^{c_{\phi}(t+u) \psi(x)} \\
& \leq \frac{2 u e^{t+u}}{e \delta_{*} c_{\phi}}
\end{aligned}
$$

which completes the proof, see (3.14), (3.11) and (3.18).

Lemma 3.8. Let $\phi, t$ and $u$ be as in Lemma 3.7. Then there exists $C_{\phi}>0$ such that, for all $\gamma \in \Gamma^{\psi}$, the following holds

$$
\left|\left(L F^{\phi_{t+u}}\right)(\gamma)-\left(L F^{\phi_{t}}\right)(\gamma)\right| \leq C_{\phi} u e^{2(t+u)} .
$$

Proof. As in (3.18), for fixed $t$ and $u$ we have

$$
\begin{aligned}
& \left|\left(L F^{\phi_{t+u}}\right)(\gamma)-\left(L F^{\phi_{t}}\right)(\gamma)\right| \leq K_{1}(\gamma)+K_{2}(\gamma)+K_{3}(\gamma), \\
& K_{1}(\gamma):=\sum_{x \in \gamma}\left|F^{\phi_{t+u}}(\gamma \backslash x)-F^{\phi_{t}}(\gamma \backslash x)\right|\left|\left(\Phi \phi_{t+u}\right)(x)-\phi_{t+u}(x)\right|, \\
& K_{2}(\gamma):=\sum_{x \in \gamma} F^{\phi_{t}}(\gamma \backslash x)\left|\left(\Phi \phi_{t+u}\right)(x)-\left(\Phi \phi_{t}\right)(x)\right|, \\
& K_{3}(\gamma):=\sum_{x \in \gamma} F^{\phi_{t}}(\gamma \backslash x)\left|\phi_{t+u}(x)-\phi_{t}(x)\right| .
\end{aligned}
$$


By (3.3) and (3.9) we have

$$
\frac{1}{1-c_{\phi}(t+u) \psi(x)} \leq \frac{1}{1-c_{\phi} \psi(x)} \leq \frac{1}{1-\psi(x)} \leq \frac{1}{\delta_{*}} .
$$

Then proceeding as in obtaining the second inequality in (3.21), we arrive at

$$
\begin{aligned}
& \left|F^{\phi_{t+u}}(\gamma \backslash x)-F^{\phi_{t}}(\gamma \backslash x)\right| \leq \frac{2 u}{\delta_{*}} \Psi(\gamma \backslash x) \prod_{y \in \gamma \backslash x}\left(1-c_{\phi}(t+u) \psi(y)\right) \\
& \quad \leq \frac{2 u}{\delta_{*}^{2}} \Psi(\gamma) \prod_{y \in \gamma}\left(1-c_{\phi}(t+u) \psi(y)\right)
\end{aligned}
$$

Next, by (3.2) and (3.3) we have

$$
\left|\left(\Phi \phi_{t+u}\right)(x)-\phi_{t+u}(x)\right| \leq\left|1-\left(\Phi \phi_{t+u}\right)(x)\right|+\left|1-\phi_{t+u}(x)\right| \leq 2 \psi(x) .
$$

We use the latter estimate and (3.24) to obtain

$$
\begin{aligned}
K_{1}(\gamma) & \leq \frac{4 u}{\delta_{*}^{2}} \Psi^{2}(\gamma) \prod_{y \in \gamma}\left(1-c_{\phi}(t+u) \psi(y)\right) \\
& \leq \frac{16 u}{\left(e \delta_{*} c_{\phi}(t+u)\right)^{2}} \prod_{y \in \gamma}\left(1-c_{\phi}(t+u) \psi(y)\right) e^{c_{\phi}(t+u) \psi(y)} \\
& \leq \frac{16 u}{\left(e \delta_{*} c_{\phi}\right)^{2}} e^{2(t+u)}
\end{aligned}
$$

By (3.14) we have

$$
\begin{aligned}
K_{2}(\gamma) & \leq \frac{1}{\delta_{*}} F^{\phi_{t}}(\gamma) \sum_{x \in \gamma}\left|\left(\Phi \phi_{t+u}\right)(x)-\left(\Phi \phi_{t}\right)(x)\right| \\
& \leq \frac{2 u n_{*} m}{\delta_{*}} \Psi(\gamma) F^{\phi_{t}}(\gamma) \leq \frac{2 u n_{*} m}{e \delta_{*} c_{\phi}} e^{t} .
\end{aligned}
$$

Similarly,

$$
K_{3}(\gamma) \leq \frac{1}{\delta_{*}} F^{\phi_{t}}(\gamma) \sum_{x \in \gamma}\left|\phi_{t+u}(x)-\phi_{t}(x)\right| \leq \frac{2 u}{e \delta_{*} c_{\phi}} e^{t} .
$$

Now we use (3.25), (3.26), (3.27) in (3.23), and thus obtain (3.22) with

$$
C_{\phi}=\frac{2 u\left(n_{*} m+1\right)}{e \delta_{*} c_{\phi}}+\frac{16 u}{\left(e \delta_{*} c_{\phi}\right)^{2}}
$$

which completes the proof.

3.3. Solving the Kolmogorov equation. Now we can turn to solving (1.2) and preparing to solving the main equation (1.3). Set

$$
E^{0}\left(\Gamma^{\psi}\right):=1 . s .\left\{F^{\phi}: \phi \in C_{\psi}(X)\right\},
$$

where 1.s. = linear span. It is a subset of the Banach space $C_{\mathrm{b}}\left(\Gamma^{\psi}\right)$ equipped with the norm

$$
\|F\|:=\sup _{\gamma \in \Gamma^{\psi}}|F(\gamma)| .
$$

Remark 3.9. The set $E^{0}\left(\Gamma^{\psi}\right)$ has all the properties stated in Proposition 2.4. This follows by the fact that the family $\left\{G^{v}: v \in \mathcal{V}\right\}$ mentioned therein is a subset of $E^{0}\left(\Gamma^{\psi}\right)$, see (3.16). In particular, it contains constant functions and each measurable and bounded $F: \Gamma^{\psi} \rightarrow \mathbb{R}$ can be obtained as the bp-limit of a sequence of the elements of $E^{0}\left(\Gamma^{\psi}\right)$. 
By (3.17) we know that $L: E^{0}\left(\Gamma^{\psi}\right) \rightarrow C_{\psi}(X)$. In view $\mathrm{f}$ this, we introduce

$$
\|F\|_{L}=\|F\|+\|L F\|, \quad F \in E^{0}\left(\Gamma^{\psi}\right),
$$

that is, $\|\cdot\|_{L}$ is the corresponding graph-norm. Thereby, we define

$$
\mathcal{D}(L)={\overline{E^{0}\left(\Gamma^{\psi}\right)}}^{L},
$$

i.e. $\mathcal{D}(L)$ is the closure of $E^{0}\left(\Gamma^{\psi}\right)$ in the graph-norm, and thus the operator $(L, \mathcal{D}(L))$ is closed. Below - in particular, in (1.2) - by $L$ we will mean this operator.

Following [1, page 108] by a classical solution of (1.2) we will understand a continuously differentiable map $\mathbb{R}_{+} \ni t \mapsto F_{t} \in \mathcal{D}(L) \subset C_{+} \mathrm{b}\left(\Gamma^{\psi}\right)$ such that both equalities in (1.2) are satisfied.

Lemma 3.10. For each $\phi \in C_{\psi}(X)$, the map $t \mapsto F^{\phi_{t}}$ is a classical solution of the Cauchy problem in (1.2) with $F_{0}=F^{\phi}$. For $n_{*}<1$, this solution satisfies $F_{t}(\gamma) \rightarrow F_{\infty}(\gamma)$ as $t \rightarrow+\infty$, where $F_{\infty}(\gamma) \equiv 1$ and the convergence is to hold for all $\gamma \in \Gamma^{\psi}$.

Proof. We begin by noting that the map $t \mapsto F^{\phi_{t}}$ has the flow property related to (3.13) and remarking that (3.17) implies

$$
\left\|L F^{\phi_{t}}\right\| \leq \frac{2}{e \delta_{*} c_{\phi}} e^{t}
$$

see (3.11) and (3.9). By (3.22) we know that the map $t \mapsto L F^{\phi t} \in C_{\mathrm{b}}\left(\Gamma^{\psi}\right)$ is continuous and hence Bochner-integrable on each interval $[a, b] \subset \mathbb{R}_{+}$. Therefore,

$$
\int_{0}^{t} L F^{\phi_{u}} d u \in C_{\mathrm{b}}\left(\Gamma^{\psi}\right)
$$

and its value at $\gamma \in \Gamma^{\psi}$ satisfies

$$
\int_{0}^{t} L F^{\phi_{u}}(\gamma) d u=F^{\phi_{t}}(\gamma)-F^{\phi}(\gamma)
$$

that readily follows by Corollary 3.6. In view of the aforementioned flow property, the proof of the first part of this statement can be done by showing that

$$
\kappa_{\phi}(t):=\left\|\frac{1}{t}\left(F^{\phi_{t}}-F^{\phi}\right)-L F^{\phi}\right\| \rightarrow 0, \quad t \rightarrow 0 .
$$

By (3.32) and (3.22) for $t \in(0,1)$ we have

$$
\begin{aligned}
& \kappa_{\phi}(t)=\sup _{\gamma \in \Gamma^{\psi}}\left|\frac{1}{t}\left(F^{\phi_{t}}(\gamma)-F^{\phi}(\gamma)\right)-L F^{\phi}(\gamma)\right| \\
\leq & \frac{1}{t} \sup _{\gamma \in \Gamma^{\psi}} \int_{0}^{t}\left|L F^{\phi_{u}}(\gamma)-L F^{\phi}(\gamma)\right| d u \leq\left(e^{2} C_{\phi} / 2\right) t,
\end{aligned}
$$

which yields (3.33). In the subcritical case $n_{*}<1$, the stated convergence follows by the concluding part of Lemma 3.2 .

Since the map $t \mapsto F^{\phi_{t}} \in C_{\mathrm{b}}\left(\Gamma^{\psi}\right)$ is continuous and bounded (by one), the Bochner integral

$$
F_{\lambda}^{\phi}=\int_{0}^{+\infty} e^{-\lambda t} F^{\phi t} d t, \quad \phi \in C_{\psi}(X),
$$

exists for all $\lambda>0$. In view of (3.22) and (3.31) , the map $t \mapsto L F^{\phi_{t}} \in C_{\mathrm{b}}\left(\Gamma^{\psi}\right)$ is continuous and absolutely $e^{-\lambda t} d t$-integrable for all $\lambda>1$. This observation leads us to the following fact. 
Lemma 3.11. For each $\phi \in C_{\psi}(X)$ and $\lambda>1$, it follows that $F_{\lambda}^{\phi}$ defined in 3.34 lies in $\mathcal{D}(L)$ and the following holds

$$
L F_{\lambda}^{\phi}=\int_{0}^{+\infty} e^{-\lambda t} L F^{\phi t} d t=-F^{\phi}+\lambda F_{\lambda}^{\phi} .
$$

Proof. In view of the existence of the Laplace transforms just discussed, the facts that $L$ is closed and $F^{\phi_{t}}$ solves the Cauchy problem in (1.2), see Lemma 3.10, both stated properties follow by a direct application of [1, Theorem 3.1.3, page 109].

Now we define

$$
\mathcal{F}^{0}\left(\Gamma^{\psi}\right)=\text { l.s. }\left\{F_{\lambda}^{\phi}: \phi \in C_{\psi}(X), \lambda>1\right\}, \quad \mathcal{F}\left(\Gamma^{\psi}\right)={\overline{\mathcal{F}^{0}\left(\Gamma^{\psi}\right)}}^{L},
$$

where $F_{\lambda}^{\phi}$ are defined in (3.34) and the closure is taken in the graph-norm (3.29).

Lemma 3.12. It follows that $\mathcal{F}\left(\Gamma^{\psi}\right)=\mathcal{D}(L)$. Thereby, $\mathcal{F}^{0}\left(\Gamma^{\psi}\right)$ is a core of $\mathcal{D}(L)$.

Proof. We begin by showing that

$$
E^{0}\left(\Gamma^{\psi}\right) \subset \mathcal{F}\left(\Gamma^{\psi}\right)
$$

i.e., each $F^{\phi}, \phi \in C_{\psi}(X)$, can be obtained as the $\|\cdot\|_{L}$-limit of a sequence of the elements of $\mathcal{F}^{0}\left(\Gamma^{\psi}\right)$ ). In fact, we are going to show that

$$
\left\|\lambda F_{\lambda}^{\phi}-F^{\phi}\right\|_{L} \rightarrow 0, \quad \text { as } \lambda \rightarrow+\infty .
$$

To this end, with the help of the first equality in (3.35) for $\lambda>1$ we write

$$
\begin{gathered}
\left|\lambda\left(L F_{\lambda}^{\phi}\right)(\gamma)-\left(L F^{\phi}\right)(\gamma)\right|=\left|\int_{0}^{+\infty}\left[\left(L F^{\phi_{t}}\right)(\gamma)-\left(L F^{\phi}\right)(\gamma)\right] e^{-\lambda t} \lambda d t\right| \\
\leq \int_{0}^{+\infty}\left|\left(L F^{\phi_{\epsilon s}}\right)(\gamma)-\left(L F^{\phi}\right)(\gamma)\right| e^{-s} d s, \quad \epsilon=1 / \lambda .
\end{gathered}
$$

Now we use here (3.22) with $t=0, u=\epsilon s$ and obtain for $\epsilon<1 / 2$ the following estimate

$$
\operatorname{LHS}\left(\underline{3.39)} \leq \epsilon C_{\phi} \int_{0}^{+\infty} s e^{-s(1-2 \epsilon)} d s=\frac{\epsilon}{(1-2 \epsilon)^{2}} C_{\phi} \rightarrow 0, \text { as } \epsilon \rightarrow 0 .\right.
$$

Next, by (3.35) - and then by (3.17) - we get

$$
\begin{aligned}
& \left\|\lambda F_{\lambda}^{\phi}-F^{\phi}\right\|=\left\|L F_{\lambda}^{\phi}\right\| \leq \int_{0}^{+\infty}\left\|L F^{\phi t}\right\| e^{-\lambda t} d t \\
& \leq \frac{2}{e \delta_{*} c_{\phi}} \int_{0}^{+\infty} e^{-(\lambda-1) t} d t=\frac{1}{\lambda-1}\left(\frac{2}{e \delta_{*} c_{\phi}}\right),
\end{aligned}
$$

where we have used the fact that $c_{\phi_{t}}=c_{\phi}(t)=c_{\phi} e^{-t}$, see (3.9). Then (3.38) readily follows by (3.40) and (3.41). Now by (3.30), (3.36) and (3.37) we get $\mathcal{D}(L) \subset \mathcal{F}\left(\Gamma^{\psi}\right)$. At the same time, by Lemma 3.11 it follows that $\mathcal{F}^{0}\left(\Gamma^{\psi}\right) \subset \mathcal{D}(L)$, which yields the opposite inclusion $\mathcal{D}(L) \supset \mathcal{F}\left(\Gamma^{\psi}\right)$.

For $\phi \in C_{\psi}(X)$ and $t \geq 0, \phi_{t}$ is also in $C_{\psi}(X)$, see Remark 3.3, and thus we can consider

$$
F_{\lambda}^{\phi_{t}}=F_{\lambda}^{\rho_{t}(\phi)}=\int_{0}^{+\infty} F^{\phi_{t}+s} e^{-\lambda s} d s
$$

and apply here Lemma 3.11 to $F_{\lambda}^{\phi_{t}}$. Then we obtain the following

Corollary 3.13. For each $\phi \in C_{\psi}(X)$ and $\lambda>1$, the map $t \mapsto F_{t}=F_{\lambda}^{\phi_{t}} \in \mathcal{D}(L) \subset$ $C_{\mathrm{b}}\left(\Gamma^{\psi}\right)$ is a classical solution of the Cauchy problem (1.2) with $F_{0}=F_{\lambda}^{\phi}$. 
Proof. Set

$$
V_{\lambda}(t)=F_{\lambda}^{\phi_{t}}-F_{\lambda}^{\phi}-t L F_{\lambda}^{\phi} .
$$

Then we apply here repeatedly (3.35) and

$$
\begin{aligned}
& V_{\lambda}(t)=\int_{0}^{t}\left(L F_{\lambda}^{\phi_{u}}-L F_{\lambda}^{\phi}\right) d u=-\int_{0}^{t}\left(F^{\phi_{u}}-F^{\phi}\right) d u+\lambda \int_{0}^{t}\left(F_{\lambda}^{\phi_{u}}-F_{\lambda}^{\phi}\right) d u \\
& =-\int_{0}^{t}\left(F^{\phi_{u}}-F^{\phi}\right) d u-\frac{\lambda t^{2}}{2} F^{\phi}+\lambda^{2} \int_{0}^{t} \int_{0}^{u} F_{\lambda}^{\phi_{v}} d u d v=: V_{\lambda}^{(1)}(t)+V_{\lambda}^{(2)}(t)+V_{\lambda}^{(3)}(t) .
\end{aligned}
$$

By Lemma 3.7 and we have that

$$
\left\|V_{\lambda}^{(1)}(t)\right\| \leq \frac{t^{2}}{\delta_{*} c_{\phi}}, \quad\left\|V_{\lambda}^{(2)}(t)\right\| \leq \frac{\lambda t^{2}}{2} .
$$

Here we recall that $F^{\phi}(\gamma) \leq 1$ for each $\phi \in C_{\psi}(X)$ and $\gamma \in \Gamma^{\psi}$, see (3.19). By this and (3.34) we also have

$$
\left\|V_{\lambda}^{(3)}(t)\right\| \leq \frac{\lambda t^{2}}{2} .
$$

We apply this estimates in (3.43) and (3.42) and then obtain

$$
\left\|V_{\lambda}(t)\right\| / t \rightarrow 0, \quad t \rightarrow 0,
$$

which completes the proof.

\section{The Fokker-Planck Equation}

4.1. Solving the Fokker-Planck equation. Now we may turn to the probabilistic part of the topic. Recall that we use probability measures on $\Gamma^{\psi}$ as states of the studied system of branching particles.

Definition 4.1. By a solution of the Fokker-Planck equation (1.3) we understand a map $\mathbb{R}_{+} \ni t \mapsto \mu_{t} \in \mathcal{P}\left(\Gamma^{\psi}\right)$ possessing the following properties: (a) for each $F \in B_{\mathrm{b}}\left(\Gamma^{\psi}\right)$, the map $\mathbb{R}_{+} \ni t \mapsto \mu_{t}(F) \in \mathbb{R}$ is measurable; (b) the equality in (1.3) holds for all $F \in \mathcal{D}(L)$, where the latter is defined in (3.30).

Theorem 4.2. For each $\mu_{0} \in \mathcal{P}\left(\Gamma^{\psi}\right)$, the Fokker-Planck equation (1.3) has a unique solution in the sense of the definition given above. Moreover, this solution is weakly continuous, i.e., $\mu_{t} \Rightarrow \mu_{s}$ as $t \rightarrow s \in \mathbb{R}_{+}$. In the subcritical case $n_{*}<1, \mu_{t} \Rightarrow \mu_{\infty}$ as $t \rightarrow+\infty$, where $\mu_{\infty}$ is the measure supported on the singleton subset of $\Gamma^{\psi}$ consisting of the empty configuration, i.e., $\mu_{\infty}\left(\Gamma^{0}\right)=1$

The proof of this theorem is based, in particular, on the following fact.

Lemma 4.3. Let a map $t \mapsto \mu_{t}$ satisfy condition (b) of Definition 4.1. Then it also satisfies (a), and hence is a solution of (1.3).

The proof of this statement in turn is based on the following result, which has its own value.

Proposition 4.4. Let $t \mapsto \mu_{t} \in \mathcal{P}\left(\Gamma^{\psi}\right)$ satisfy (1.3) for all $t_{1}, t_{2}$ and $F \in \mathcal{D}(L)$. Then, for each $F \in \mathcal{F}^{0}\left(\Gamma^{\psi}\right)$, the map $t \mapsto \mu_{t}(F) \in \mathbb{R}$ is Lipschitz-continuous. The same is true also for $F \in E^{0}\left(\Gamma^{\psi}\right)$, see (3.28).

Proof. First, we rewrite (1.3) in the form

$$
\mu_{t_{2}}(F)=\mu_{t_{1}}(F)+\int_{t_{1}}^{t_{2}} \mu_{s}(L F) d s, \quad 0 \leq t_{1}<t_{2} .
$$


Take $F=F_{\lambda}^{\phi}, \phi \in C_{\psi}(X), \lambda>1$. By (3.16), and then by (3.34) and (3.35), we have $\left\|L F_{\lambda}^{\phi}\right\| \leq 2$. Then by (4.1) one obtains

$$
\left|\mu_{t_{2}}\left(F_{\lambda}^{\phi}\right)-\mu_{t_{1}}\left(F_{\lambda}^{\phi}\right)\right| \leq 2\left|t_{2}-t_{1}\right| .
$$

For $F=\sum_{n} \alpha_{n} F_{\lambda_{n}}^{\phi_{n}} \in \mathcal{D}^{0}(L)$, this yields

$$
\left|\mu_{t_{2}}(F)-\mu_{t_{1}}(F)\right| \leq 2\left(\sum_{n}\left|\alpha_{n}\right|\right)\left|t_{2}-t_{1}\right| .
$$

Now for $F=F^{\phi}, \phi \in C_{\psi}(X)$, see (3.28), (3.30), by (3.17) we have

$$
\left|\mu_{t_{2}}\left(F^{\phi}\right)-\mu_{t_{1}}\left(F^{\phi}\right)\right| \leq \frac{2}{e \delta_{*} c_{\phi}}\left|t_{2}-t_{1}\right| .
$$

The extension of the latter to the linear combinations of $F^{\phi_{n}}$ can be done similarly as above.

Proof of Lemma 4.3. By Remark 3.9 we know that $E^{0}\left(\Gamma^{\psi}\right)$ is $b p$-dense in $B_{\mathrm{b}}\left(\Gamma^{\psi}\right)$. Then the measurability of $t \mapsto \mu_{t}(F), F \in B_{\mathrm{b}}\left(\Gamma^{\psi}\right)$ follows by the continuity (hence, measurability) just proved.

Proof of Theorem 4.2. In view of the lemma just proved, it remains to establish the existence and uniqueness of solutions of (1.3) with $F \in \mathcal{D}(L)$. First we prove existence. Let $F$ be in $\mathcal{F}^{0}\left(\Gamma^{\psi}\right)$ which is the core of $\mathcal{D}(L)$, see (3.36). Since (1.3) is linear, it is enough to take $F=F_{\lambda}^{\phi}$ with $\phi \in C_{\psi}(X)$ and $\lambda>1$. For a given $\mu \in \mathcal{P}\left(\Gamma^{\psi}\right)$ and $t>0$, we then set

$$
\mu_{t}\left(F_{\lambda}^{\phi}\right)=\mu\left(F_{\lambda}^{\phi_{t}}\right)
$$

which has to hold for all $\phi \in C_{\psi}(X)$ and $\lambda>1$. Let us multiply both sides of (4.2) by $\lambda$ and then pass to the limit $\lambda \rightarrow+\infty$. By (3.38) we then get that $\mu_{t}\left(F^{\phi}\right)=\mu\left(F^{\phi_{t}}\right)$, which uniquely determines $\mu_{t}$ in view of property (iii) of Proposition 2.4 and the uniqueness stated in Lemma 3.2, see also Remark 3.9. Note that $\mu_{t}\left(F^{\phi}\right) \in(0,1)$ for all $t>0$. Then by (3.35) we have

$$
\begin{gathered}
\int_{t_{1}}^{t_{2}} \mu_{s}\left(L F_{\lambda}^{\phi}\right) d s=-\int_{t_{1}}^{t_{2}} \mu_{s}\left(F^{\phi}\right) d s+\int_{t_{1}}^{t_{2}} \mu_{s}\left(\lambda F_{\lambda}^{\phi}\right) d s \\
=-\int_{t_{1}}^{t_{2}} \mu_{s}\left(F^{\phi}\right) d s+\int_{t_{1}}^{t_{2}} \int_{0}^{+\infty} \lambda e^{-\lambda t} \mu_{s}\left(F^{\phi t}\right) d s d t
\end{gathered}
$$

where we used also Fubini's theorem. Thereafter, by (4.2) and the flow property, see (3.13), we get $\mu_{s}\left(F^{\phi_{t}}\right)=\mu_{s+t}\left(F^{\phi}\right)$ and then use this in the second summand (name it $\Upsilon$ ) of the last line of (4.3), then integrate by parts and by Fubini's theorem obtain

$$
\begin{gathered}
\Upsilon=\int_{t_{1}}^{t_{2}} \mu_{s}\left(F^{\phi}\right) d s+\int_{t_{1}}^{t_{2}} \frac{d}{d s}\left(\int_{0}^{+\infty} e^{-\lambda t} \mu_{s+t}\left(F^{\phi}\right) d t\right) d s \\
=\int_{t_{1}}^{t_{2}} \mu_{s}\left(F^{\phi}\right) d s+\int_{t_{1}}^{t_{2}} \frac{d}{d s} \mu_{s}\left(\int_{0}^{+\infty} e^{-\lambda t} F^{\phi_{t}} d t\right) d s \\
=\int_{t_{1}}^{t_{2}} \mu_{s}\left(F^{\phi}\right) d s+\int_{t_{1}}^{t_{2}} \frac{d}{d s} \mu_{s}\left(F_{\lambda}^{\phi}\right) d s \\
=\int_{t_{1}}^{t_{2}} \mu_{s}\left(F^{\phi}\right) d s+\mu_{t_{2}}\left(F_{\lambda}^{\phi}\right)-\mu_{t_{1}}\left(F_{\lambda}^{\phi}\right) .
\end{gathered}
$$


Now we plug this in (4.3) and get that the map $t \mapsto \mu_{t}(F), F \in \mathcal{F}^{0}\left(\Gamma^{\psi}\right)$, solves (4.1). For $F \in \mathcal{D}(L)$, let $\left\{F_{n}\right\}_{n \in \mathbb{N}} \subset \mathcal{F}^{0}\left(\Gamma^{\psi}\right)$ be such that $\left\|F-F_{n}\right\|_{L} \rightarrow 0$ as $n \rightarrow+\infty$. Then

$$
\begin{aligned}
\mid \mu_{t_{2}}(F) & -\mu_{t_{1}}(F)-\int_{t_{1}}^{t_{2}} \mu_{s}(L F) d s|\leq| \mu_{t_{2}}\left(F-F_{n}\right)|+| \mu_{t_{1}}\left(F-F_{n}\right) \mid \\
& +\int_{t_{1}}^{t_{2}}\left|\mu_{s}\left(L F-L F_{n}\right)\right| d s \leq\left(t_{2}-t_{1}+2\right)\left\|F-F_{n}\right\|_{L},
\end{aligned}
$$

which yields that $t \mapsto \mu_{t}(F), F \in \mathcal{D}(L)$ also solves (4.1).

Assume now that $t \mapsto \tilde{\mu}_{t}$ is another solution of (1.3), and hence of (4.1), satisfying $\left.\tilde{\mu}_{t}\right|_{t=0}=\mu$. By Proposition 4.4 the map $t \mapsto \tilde{\mu}(F), F \in \mathcal{F}^{0}\left(\Gamma^{\psi}\right)$ is Lipschitz-continuous. Then, for each $\lambda>1$ and $\phi \in C_{\psi}(X)$, we have

$$
d \tilde{\mu}_{s}\left(F_{\lambda}^{\phi}\right)=\tilde{\mu}_{s}\left(L F_{\lambda}^{\phi}\right) d s
$$

holding for Lebesgue-almost all $s \geq 0$. Then

$$
\begin{gathered}
-\lambda \int_{0}^{t} e^{-\lambda s} \tilde{\mu}_{s}\left(F_{\lambda}^{\phi}\right) d s=\int_{0}^{t} \tilde{\mu}_{s}\left(F_{\lambda}^{\phi}\right) d e^{-\lambda s} \\
=\tilde{\mu}_{t}\left(F_{\lambda}^{\phi}\right) e^{-\lambda t}-\tilde{\mu}_{0}\left(F_{\lambda}^{\phi}\right)-\int_{0}^{t} e^{-\lambda s} \tilde{\mu}_{s}\left(L F_{\lambda}^{\phi}\right) d s \\
=\tilde{\mu}_{t}\left(F_{\lambda}^{\phi}\right) e^{-\lambda t}-\tilde{\mu}_{0}\left(F_{\lambda}^{\phi}\right)-\lambda \int_{0}^{t} e^{-\lambda s} \tilde{\mu}_{s}\left(F_{\lambda}^{\phi}\right) d s+\int_{0}^{t} e^{-\lambda s} \tilde{\mu}_{s}\left(F^{\phi}\right) d s .
\end{gathered}
$$

This yields

$$
\mu\left(F_{\lambda}^{\phi}\right)=\tilde{\mu}_{0}\left(F_{\lambda}^{\phi}\right)=\tilde{\mu}_{t}\left(F_{\lambda}^{\phi}\right) e^{-\lambda t}+\int_{0}^{t} e^{-\lambda s} \tilde{\mu}_{s}\left(F^{\phi}\right) d s, \quad \lambda>1,
$$

which after passing to the limit $t \rightarrow+\infty$ leads to

$$
\mu\left(F_{\lambda}^{\phi}\right)=\int_{0}^{+\infty} e^{-\lambda s} \tilde{\mu}_{s}\left(F^{\phi}\right) d s
$$

that holds for all $\lambda>1$. By the very definition in (4.2) the map $t \mapsto \mu_{t}\left(F^{\phi}\right)$ is continuous; the continuity of $t \mapsto \tilde{\mu}_{t}\left(F^{\phi}\right)$ was established in Proposition 4.4. Both maps are bounded. By (3.34) and (4.2), and then by (4.4), the Laplace transforms of both these maps coincide. Therefore, by Lerch's theorem $\mu_{t}\left(F^{\phi}\right)=\tilde{\mu}_{t}\left(F^{\phi}\right)$ for all $t>0$ and $\phi \in C_{\psi}(X)$. As mentioned above, see Proposition 2.4, the class of functions $\left\{F^{\phi}: \phi \in C_{\psi}(X)\right\}$ is separating, that means $\mu_{t}=\tilde{\mu}_{t}, t>0$ and hence the stated uniqueness. The proof the weak convergence $\mu_{t} \Rightarrow \mu_{s}$ follows by (4.2) and the fact that $\left\{F^{\phi}: \phi \in C_{\psi}(X)\right\}$ is also convergence determining, see again Proposition 2.4. It remains to prove that $\mu_{t} \Rightarrow \mu_{\infty}$ as $t \rightarrow+\infty$. Since the set $\left\{F^{\phi}: \phi \in C_{\psi}(X)\right\}$ is convergence determining, to this end it is enough to show that $\mu_{t}\left(F^{\phi}\right) \rightarrow \mu_{\infty}\left(F^{\phi}\right)=1$, holding for all $\phi \in C_{\psi}(X)$. By (4.2) and the concluding statement of Lemma 3.10 we have

$$
\lim _{t \rightarrow+\infty} \mu_{t}\left(F^{\phi}\right)=\lim _{t \rightarrow+\infty} \mu\left(F^{\phi_{t}}\right)=\mu\left(F_{\infty}\right)=1,
$$

which completes the whole proof.

4.2. Concluding comments. As mentioned above, our main aim in this work is to find a way of describing branching in infinite particle systems. That is why we restrict ourselves to the results stated in Theorem 4.2, A direct consequence of Theorem 4.2 is the existence of a Markov process with values in $\Gamma^{\psi}$, that may be constructed by means 
of the Markov transition function $p_{\gamma}^{t}$, see [12, pages 156, 157], determined by its values on $\left\{F^{\phi}: \phi \in C_{\psi}(X)\right\}$, cf. Remark 3.9. These values are given by the following formula

$$
p_{t}^{\gamma}\left(F^{\theta}\right)=F^{\phi_{t}}(\gamma), \quad \gamma \in \Gamma^{\psi} .
$$

It definitely has the branching property, cf. [19, page 29],

$$
F^{\phi_{t}}\left(\gamma_{1} \cup \gamma_{2}\right)=F^{\phi_{t}}\left(\gamma_{1}\right) F^{\phi_{t}}\left(\gamma_{2}\right), \quad \gamma_{1}, \gamma_{2} \in \Gamma^{\psi} .
$$

Then in accord with the definition on page 30 of [19, the aforementioned Markov process would be a measure-valued branching process. The uniqueness stated in Theorem 4.2 can be used to prove that such a process is unique up to modifications. Another observation is that, in our model, branching is the only evolutionary act, whereas papers on branching in finite particle systems, e.g., [2, 3, 9, 11], assume more such acts, e.g., diffusion in $X$. These and similar generalizations can also be done in our setting.

\section{ACKNOWLEDGEMENTS}

The research of the first named author was financially supported by National Science Centre, Poland, grant 2017/25/B/ST1/00051, that is acknowledged by him. The authors are cordially grateful to both referees for their careful reading of the manuscript, constructive criticism and valuable and favorable suggestions that helped to improve the quality of this work.

\section{REFERENCES}

[1] W. Arendt, Ch. J. K. Batty, M. Hieber, F. Neubrander, Vector-valued Laplace Transforms and Cauchy Problems, Second Edition, Monographs in Mathematics, Vol. 96, Birkhäser, Basel, 2011.

[2] L. Beznea, O. Lupaşcu, Measure-valued discrete branching Markov processes, Trans. Amer. Math. Soc. 368 (2016), 5153-5176.

[3] L. Beznea, O. Lupaşcu-Stamate, C. I. Vrabie, Stochastic solutions to evolution equations of non-local branching processes, Nonlinear Anal. 200 (2020) 112021.

[4] L. Beznea, M. Röckner, From resolvents to cadlag processes through compact excessive functions and applications to singular SDE on Hilbert spaces, Bull. Sci. math. 135 (2011) 844-870.

[5] V. I. Bogachev, N. V. Krylov, M. Röckner, S. V. Shaposhnikov, Fokker-Planck-Kolmogorov Equations. Mathematical Surveys and Monographs, 207. American Mathematical Society, Providence, RI, 2015.

[6] D. J. Daley, D. Vere-Jones, An Introduction to the Theory of Point Processes. Vol. II. General Theory and Structure. Second edition. Probability and its Applications (New York). Springer-Verlag, New York, 2008.

[7] D. L. Cohn, Measure Theory. Second edition. Birkhäuser Advanced Texts: Basler Lehrbücher. Birkhäuser/Springer, New York, 2013.

[8] D. A. Dawson, Measure-Valued Markov Processes. École d'Été de Probabilités de Saint-Flour XXI1991, 1-260, Lecture Notes in Math., 1541, Springer, Berlin, 1993.

[9] D. A. Dawson, L. G. Gorostiza, Z. Li, Nonlocal branching superprocesses and some related models, Acta Applicandae Mathematicae 74 (2002), 93-112.

[10] R. L. Dobrushin, Y. G. Sinai, Y. M. Sukhov, Dynamical systems of statistical mechanics, in Dynamical Systems II. Encyclopaedia of Mathematical Sciences, vol 2., Y. G. Sinai, eds, Springer, Berlin, Heidelberg, 1989.

[11] E. B. Dynkin, S. E. Kuznetsov, A. V. Skorokhod, Branching measure-valued processes, Probab. Theory Relat, Fields 99 (1994), 55-96.

[12] S. N. Ethier, T. G. Kurtz, Markov Processes: Characterization and Convergence, Wiley, New York, 1986.

[13] V. Konarovskyi, A system of coalescing heavy diffusion particles on the real line, Ann. Probab. 45 (2017), 3293-3335.

[14] V. Konarovskyi, M. von Renesse, Modified massive Arratia flow and Wasserstein diffusion, Commun. Pure and Appl. Math. 72 (2019), 764-800.

[15] Y. Kozitsky, Stochastic branching at the edge: Individual-based modeling of tumor cell proliferation, J. Evol. Equ. 21 (2021), 2081-2104.

[16] Yu. Kozitsky, K. Pilorz, Random jumps and coalescence in the continuum: evolution of ststes of an infinite particle system, Discrete Cont. Dyn-A 40 (2020), 725-752. 
[17] Y. Kozitsky, M. Röckner, A Markov process for an infinite interacting particle system in the continuum, Electron. J. Probab. 26 (2021), article no. 72, 1-53. .

[18] A. Lenard, Correletion functions and the uniqueness of the state in classical statistical mechanics, Comm. Math. Phys. 30 (1973), 35-44.

[19] Z. Li, Measure-Valued Branching Markov Processes, Probability and its Applications, Springer, Heidelberg Dordrecht London New York, 2011.

[20] Z.-M. Ma, M. Röckner, Introduction to the Theory of (Nonsymmetric) Dirichlet Forms, Universitext, Springer-Verlag Berlin Heidelberg, 1992.

[21] K. R. Parthasarathy, Probability Measures on Metric Spaces, Probability and Mathematical Statistics, No. 3 Academic Press, Inc., New York-London 1967.

[22] B. Simon, The Statistical Mechanics of Lattice Gases. I, Princeton University Press, Pronceton, NJ, 1993.

[23] H. Zessin, The method of moments for random measures, Z. Wahrscheinlichkeitstheorie verw. Gebiete. 62 (1983), 395-409.

Instytut Matematyki, Uniwersytet Mari Curie-SkŁodowskiej, 20-031 Lublin, Poland Email address: jkozi@hektor.umcs.lublin.pl

Politechnika Lubelska, 20-618 Lublin, Poland 